\title{
Speaker Verification with Adaptive Spectral Subband Centroids
}

\author{
Tomi Kinnunen ${ }^{1}$, Bingjun Zhang ${ }^{2}$, Jia Zhu ${ }^{2}$, and Ye Wang ${ }^{2}$ \\ ${ }^{1}$ Speech and Dialogue Processing Lab \\ Institution for Infocomm Research $\left(\mathrm{I}^{2} \mathrm{R}\right)$ \\ 21 Heng Mui Keng Terrace, Singapore 119613 \\ ktomi@i2r.a-star.edu.sg \\ ${ }^{2}$ Department of Computer Science \\ School of Computing, National University of Singapore (NUS) \\ 3 Science Drive 2, Singapore 117543 \\ \{bingjun, zhujia, wangye\}@comp.nus.edu.sg
}

\begin{abstract}
Spectral subband centroids (SSC) have been used as an additional feature to cepstral coefficients in speech and speaker recognition. SSCs are computed as the centroid frequencies of subbands and they capture the dominant frequencies of the short-term spectrum. In the baseline SSC method, the subband filters are pre-specified. To allow better adaptation to formant movements and other dynamic phenomena, we propose to adapt the subband filter boundaries on a frame-by-frame basis using a globally optimal scalar quantization scheme. The method has only one control parameter, the number of subbands. Speaker verification results on the NIST 2001 task indicate that the selection of the parameter is not critical and that the method does not require additional feature normalization.
\end{abstract}

\section{Introduction}

The so-called mel-frequency cepstral coefficients 1] (MFCC) have proven to be efficient feature set for speaker recognition. A known problem of cepstral features, however, is noise sensitivity. For instance, convolutive noise shifts the mean value of the cepstral distribution whereas additive noise tends to modify the variances [2]. To compensate for the feature mismatch between training and verification utterances, normalizations in feature, model and score domains are commonly used [3].

Spectral subband centroids [4; 5; 6; 7] (SSC) are an alternative to cepstral coefficients. SSCs are computed as the centroid frequencies of subband spectra and they give the locations of the local maxima of the power spectrum. SSCs have been used for speech recognition [4; 5], speaker recognition [7] and audio fingerprinting [6]. Recognition accuracy of SSCs is lower in noise-free conditions compared with MFCCs. However, SSCs can outperform MFCCs in noisy conditions and they can be combined with MFCCs to provide complementary information. 
Spectral subband centroids (SSC)

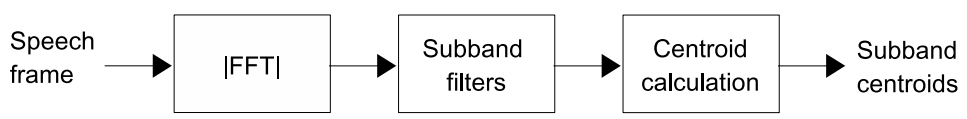

\section{Optimal scalar quantization based spectral subband centroids (OSQ-SSC)}

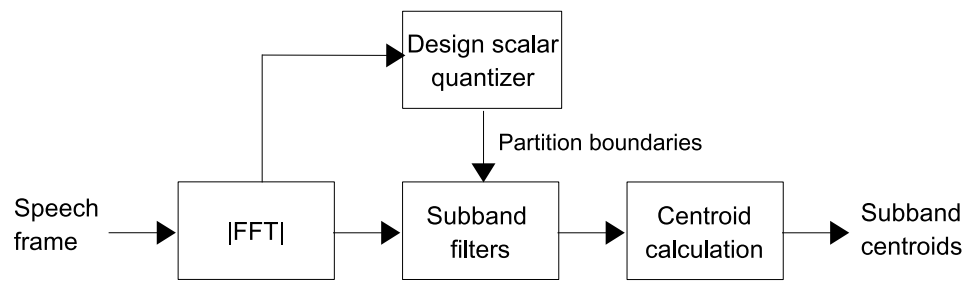

Fig. 1. Computation of the SSC and the proposed OSQ-SSC features. In SSC, the subband boundaries are fixed and in OSQ-SSC, the boundaries are re-calculated for every frame by partitioning the spectrum with optimal scalar quantization.

The key component of the SSC method is the filterbank. Design issues include the number of subbands, the cutoff frequencies of the subband filters, the shape of the subband filters, overlapping of the subband filters, compression of the spectral dynamic range and so on [5]. The parameters of the filterbank can be optimized experimentally for a given task and operating conditions.

In this study, our aim is to simplify the parameter setting of the SSC method by adding some self-adaptivity to the filterbank. In particular, we optimize the subband filter cutoff frequencies on a frame-by-frame basis to allow better adaptation to formant movements and other dynamic phenomena. We consider the subbands as partitions or quantization cells of a scalar quantizer. Each subband centroid is viewed as the representative value of that cell and the problem can be defined as joint optimization of the partitions and the centroids. The difference between the conventional SSC method and the proposed method is illustrated in Fig. 1]

\section{Spectral Subband Centroids}

In the following, we denote the FFT magnitude spectrum of a frame by $S[k]$, where $k=1, \ldots, N$ denotes the discrete frequency index. The index $k=N$ corresponds to the half sample rate $f_{s} / 2$. The $m^{\text {th }}$ subband centroid is computed as follows [5]:

$$
c_{m}=\frac{\sum_{k=q_{l}(m)}^{q_{h}(m)} k W_{m}[k] S^{\gamma}[k]}{\sum_{k=q_{l}(m)}^{q_{h}(m)} W_{m}[k] S^{\gamma}[k]},
$$

where $W_{m}[k]$ are the $m^{\text {th }}$ bandpass filter coefficients, $q_{l}(m), q_{h}(m) \in[1, N]$ are its lower and higher cutoff frequencies and $\gamma$ is a dynamic range parameter. 
The shape of the subband filter introduces bias to the centroids. For instance, the triangular shaped filters used in MFCC computation [1] shift the centroid towards the mid part of the subband. To avoid such bias, we use a uniform filter in (11): $W_{m}[k]=1$ for $q_{l}(m) \leq k \leq q_{h}(m)$. Furthermore, we set $\gamma=1$ in this study. With these modifications, (1) simplifies to

$$
c_{m}=\frac{\sum_{k=q_{l}(m)}^{q_{h}(m)} k S[k]}{\sum_{k=q_{l}(m)}^{q_{h}(m)} S[k]} .
$$

\section{Adapting the Subband Boundaries}

To allow better adaptation of the subband centroids to formant movements and other dynamic phenomena, we optimize the filter cutoff frequencies on a frameby-frame basis. We use scalar quantization as a tool to partition the magnitude spectrum into $K$ non-overlapping quantization cells. The subband cutoff frequencies, therefore, are given by the partition boundaries of the scalar quantizer.

The expected value of the squared distortion for the $m^{\text {th }}$ cell is defined as

$$
e_{m}^{2}=\sum_{q(m-1)<k \leq q(m)} p_{k}\left(k-c_{m}\right)^{2},
$$

where $p_{k}=S[k] / \sum_{n=1}^{N} S[n]$ is the normalized FFT magnitude, $c_{m}$ is the subband centroid as defined in (2) and $q(m-1), q(m)$ are the subband boundaries: $0=q(0)<q(1)<q(2)<\cdots<q(K)=N$. The scalar quantizer design can then be defined as the minimization of the total error:

$$
\min _{(q(0), q(1), \ldots, q(K))} \sum_{m=1}^{K} e_{m}^{2}
$$

The number of subbands $(K)$ is considered as a control parameter that needs to be optimized experimentally for a given application.

We have implemented a globally optimal scalar quantizer which uses matrix searching technique to solve (41) [8]. The time complexity of the method is $O(K N)$ and our implementation runs 18 times faster than realtime on a $3 \mathrm{GHz}$ Pentium processor for $(K, N)=(8,128)$. It is interesting to note that optimal algorithms for vector quantization [9] require exponential time but globally optimal scalar quantizer can be designed in polynomial time. This theoretically interesting property, in fact, was one of our initial motivations to apply the method to feature extraction. We term the proposed method as optimal scalar quantization based spectral subband centroids (OSQ-SSC).

Figure 2 shows the centroids from both the SSC with mel filterbank and the OSQ-SSC method. The spectrogram is also shown as a reference. It can be seen that the OSQ-SSC features are better adapted to local dynamic changes of the spectrum compared with SSC. In particular, the centroids from OSQ-SSC tend to follow the F0 harmonics and the formant frequencies during voiced regions. 


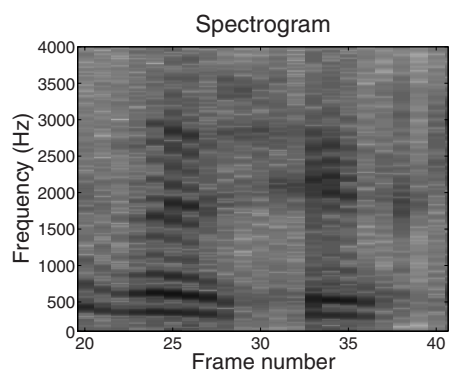

(a)

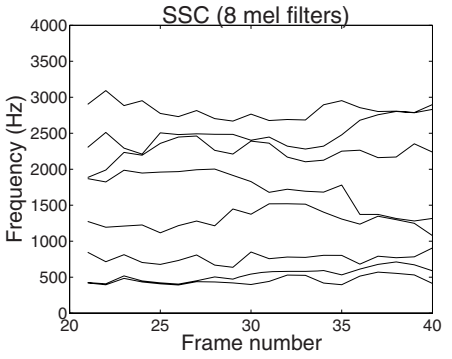

(b)

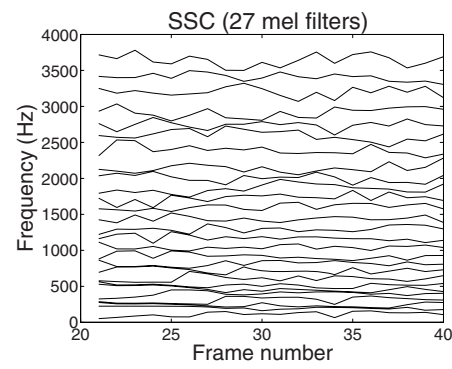

(d)

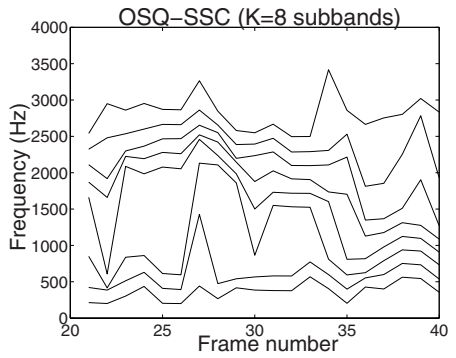

(c)

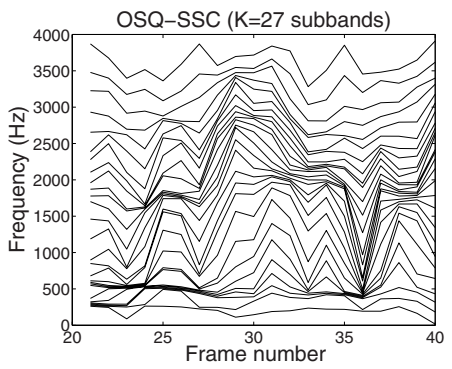

(e)

Fig. 2. Illustrations of conventional spectral subband centroids with fixed filterbank (SSC) and the proposed method with adapted subband boundaries (OSQ-SSC)

\section{Speaker Verification Setup}

We use the NIST 2001 speaker recognition evaluation corpus, a conversational cellular phone corpus, in our experiment:1. The 1-speaker detection task as defined by NIST consists of 174 target speakers and 22418 verification trials with a genuine/impostor ratio of 1:10. The amount of training data per speaker is about 2 minutes and the duration of the test segments varies from a few seconds up to one minute.

${ }^{1}$ http://wWw.nist.gov/speech/tests/spk/ 
We use the state-of-the-art Gaussian mixture model - universal background model (GMM-UBM) with diagonal covariance matrices as the recognizer [10]. The background model is trained using the development set of the NIST 2001 corpus with the expectation-maximization (EM) algorithm. Target speaker models are derived with maximum a posteriori (MAP) adaptation of the mean vectors and the verification score is computed as the average log-likelihood ratio. The GMMs have 256 Gaussian components for all the features and parameters tested.

We include the standard MFCC front-end as a reference, including $12 \mathrm{MFCC}$ with their delta and double-delta coefficients. RASTA filtering, energy-based voice activity detection (VAD) and mean/variance normalization are applied to enhance robustness. The same VAD is used with SSC and OSQ-SSC features.

\section{$5 \quad$ Speaker Verification Results}

We first study the effects of the number of subbands and feature normalization. Feature normalization is performed after voice activity detection to give zero mean and/or unit variance features. The equal error rates (EER) for the NIST 2001 evaluation set are shown in Table 1. The best result is obtained without any normalization, indicating that OSQ-SSC is a robust feature in itself (In contrast, both the mean and variance normalization were helpful for MFCC). Optimal number of subbands is $K=8$. For too few subbands, speaker discrimination is expected to be poor. For too many subbands, on the other hand, the spacing of the centroids becomes small. This makes the different frames similar to each other, removing some useful variability.

Table 1. Effects of the number subbands and normalization to accuracy of the OSQSSC feature (EER \%)

\begin{tabular}{|c|c|c|c|c|}
\hline Subbands & \multicolumn{3}{|c|}{ Feature normalization } \\
\hline & None & Mean & Var & Mean+Var \\
\hline \hline 4 & 19.4 & 27.5 & 31.7 & 27.5 \\
8 & 18.0 & 24.8 & 29.8 & 24.5 \\
16 & 19.1 & 23.1 & 27.5 & 23.3 \\
32 & 19.8 & 24.6 & 28.4 & 24.2 \\
\hline
\end{tabular}

We next compare OSQ-SSC, their delta coefficients and the concatenation of the static and delta coefficients at the frame level. Based on Table1, we turn off the normalizations. The results are given in Table 2. The delta coefficients yield higher error rates compared with the static coefficients which is expected. We did, however, expect some improvement when combining static and delta features which is not the case. The error rates of the delta coefficient are relatively high compared with the static coefficient which partly explains why the fusion is not successful. The simple differentiator method for computing deltas may not be robust enough and other methods like linear regression should be considered. 
Table 2. Comparison of static and delta features of OSQ-SSC (EER \%)

\begin{tabular}{|c|c|c|c|}
\hline Subbands & \multicolumn{3}{|c|}{ Feature set } \\
\hline & OSQ-SSC & $\Delta$ OSQ-SSC & OSQ-SSC $+\Delta$ \\
\hline \hline 4 & 19.4 & 26.9 & 20.3 \\
8 & 18.0 & 22.2 & 19.7 \\
16 & 19.1 & 23.4 & 20.3 \\
32 & 19.8 & 24.0 & 21.3 \\
\hline
\end{tabular}

Table 3. Comparison of MFCC, OSQ-SSC and $\triangle$ OSQ-SSC under additive white noise condition (EER \%)

\begin{tabular}{|c|c|c|c|}
\hline $\begin{array}{c}\text { Noise } \\
\text { weight }(\alpha)\end{array}$ & \multicolumn{3}{|c|}{ Feature set } \\
\hline \hline 0 & 8.3 & 18.0 & 22.2 \\
0.3 & 15.7 & 19.9 & 26.6 \\
0.6 & 18.4 & 22.6 & 29.2 \\
0.9 & 25.6 & 28.7 & 47.9 \\
\hline
\end{tabular}

We next study noise robustness of the OSQ-SSC feature. We contaminated all the training and testing files with additive white noise with three different noise levels. The noise was added with linear combination of the speech and noise as $x_{\text {noisy }}[n]=\alpha z[n]+(1-\alpha) x_{\text {orig }}[n]$, where $x_{\text {noisy }}[n], z[n]$ and $x_{\text {orig }}[n]$ denote the noisy speech, noise and the original speech signals, respectively. The results for $K=8$ subbands and their delta coefficients are given in Table 3. The MFCC result is shown as a reference.

All the three features degrade when noise level is increased, which is expected. The MFCC feature gives the best result in all cases and $\triangle \mathrm{OSQ}-\mathrm{SSC}$ gives the worst result in all conditions. However, relative degradation of OSQ-SSC is much smaller compared with MFCC. For instance, the relative increase in EER from $\alpha=0$ to $\alpha=0.3$ is $89 \%$ for MFCC, whereas for OSQ-SSC it is only $11 \%$. This is interesting since the MFCC features have 36 dimensions, including deltas and double deltas, mean and variance normalization and RASTA filtering. In turn, OSQ-SSC has only 8 dimensions and is without any normalizations. We interpret the result so that the intrinsic resistance to additive noise of OSQ-SSC is better than that of MFCC. On the other hand, speaker discrimination of MFCC is clearly higher.

Finally, we compare OSQ-SSC with SSC. We consider the following three filterbank configurations for the SSC feature:

- SSC(1) : linear frequency scale, non-overlapping rectangular filters

- SSC(2) : mel frequency scale, non-overlapping rectangular filters

- SSC(3) : mel frequency scale, overlapping triangular filters

According to 7], mean subtraction helps SSC. We confirmed this experimentally and we apply it in all the three cases. The results are shown in Table 4. 
Table 4. Comparison SSC and OSQ-SSC (EER \%)

\begin{tabular}{|c|c|c|c|c|}
\hline Subbands & \multicolumn{4}{|c|}{ Feature set } \\
& OSQ-SSC & SSC(1) & SSC(2) & SSC(3) \\
\hline \hline 4 & 19.4 & 24.8 & 23.9 & 24.8 \\
8 & 18.0 & 19.7 & 21.9 & 15.4 \\
16 & 19.1 & 21.0 & 25.3 & 17.5 \\
32 & 19.8 & 24.2 & 26.3 & 22.5 \\
\hline
\end{tabular}

The performance of the SSC method strongly depends on the parameter setting. The best $\mathrm{SSC}$ result $(\mathrm{EER}=15.4 \%)$ is obtained by using eight overlapping filters on the mel-scale. Overall, $\mathrm{SSC}(3)$ gives the best result among the three filterbank configurations, followed by SSC(1) and SSC(2), respectively. Overlapping filters are useful for SSC.

Comparing OSQ-SSC with SSC, OSQ-SSC is less sensitive to parameter setup. The method has only one control parameter and the results incidate that the method is not sensitive to it. For $\mathrm{SSC}(3)$, the error rate varies between $15.4 \%$ - $24.8 \%$ whereas for OSQ-SSC, the range is $18.0 \%$ - $19.8 \%$. The OSQ-SSC method has a built-in "self-optimizing" property of the filterbank. The success of the SSC method, on the other hand, depends on the correct setting of the filterbank parameters.

\section{Discussion}

The different settings for SSC presented in Table 4 indicate that SSC gives the best results when the filterbank resembles the one used with MFCC features (mel-frequency scale with triangular overlapping filters). For OSQ-SSC, this is not the case by definition (filters are rectangular and non-overlapping).

The advantage of the OSQ-SSC feature over the baseline SSC feature, in theory, is that the subband boundaries are adapted for each frame. The partitions of the scalar quantizer, however, are themselves non-overlapping. This forces the centroid frequencies to be monotonically increasing, thereby limiting their dynamic range. The results of Table 4 on the other hand, indicate the usefulness of overlapping filters. A possible future direction would be studying optimization of the filterbank parameters using a probabilistic clustering model such as the GMM.

Does the centroid information provide complementary information that is not captured by the MFCCs? Overall, the accuracies of both the SSC- and OSQSSC-based recognizers are significantly lower compared with the MFCC-based features which is disappointing. We did several pairwise fusion experiments, combining both the SSC and OSQ-SSC classifier output scores with the MFCC scores by weighted sum. None of these lead to improvement even when the fusion weights were optimized on the evaluation set. This suggests that the cepstrumand centroid-based classifiers are redundant. The centroid information seems to be already absorbed into the MFCCs. 
In [7] SSCs yielded comparable results to MFCCs in noise-free condition. Moreover, SSCs outperformed MFCCs under additive noise conditions with low signal-to-noise ratios. We did not observe similar pattern; MFCCs outperformed both the SSCs and OSQ-SSCs with a wide margin in all cases. One source for this disparity may arise from implemetation differences of the feature extraction, in particular, the channel compensation methods applied. In [7], mean subtraction was used with the SSC features, but no channel compensation was mentioned in conjunction with the MFCC features. Our MFCC front-end, on the other hand, includes RASTA filtering and utterance level mean/variance normalization to increase robustness. Our MFCC front-end is comparatively more robust and the centroid information does not seem to yield additional gain in this case.

\section{Conclusions and Future Work}

We have studied subband centroid based features for the speaker verification task. In particular, we simplified the spectral subband centroid (SSC) method by adding self-adaptivity to the filterbank. The proposed feature (OSQ-SSC) has one control parameter and the experiments indicated that the method is not sensitive to it. It was also found that the proposed feature does not require normalization like MFCC and SSC. This is beneficial for real-time applications.

Our experiments indicate that the centroid-based features have limited use in speaker verification if a robust MFCC front-end is used. The theoretical advantage of SSCs over MFCCs would be that they have a direct physical interpretation. Therefore, SSCs and/or OSQ-SSCs might be used as alternatives to traditional LPC-based formant estimation in forensic speaker recognition [11]. Another potential application would be speech recognition. The subband centroids are related to formant frequencies, and they depend, in addition to the speaker, on the text spoken. Recently SSC-based features have shown promise as an additional feature in noisy speech recognition tasks $12 ;$; .

From the theoretical side, relation of the OSQ-SSC centroid frequencies to poles of the LPC model would be an interesting future direction. The cepstral coefficients derived from the LPC model [13] have been successful in speaker verification in addition to MFCCs. The centroids given by OSQ-SSC might be an alternative, numerically stable, "pole" presentation of speech signals.

\section{References}

[1] Davis, S., Mermelstein, P.: Comparison of parametric representations for monosyllabic word recognition in continuously spoken sentences. IEEE Trans. Acoustics, Speech, and Signal Processing 28(4), 357-366 (1980)

[2] Pelecanos, J., Sridharan, S.: Feature warping for robust speaker verification. In: Proc. Speaker Odyssey: the Speaker Recognition Workshop (Odyssey 2001), Crete, Greece, pp. 213-218 (2001) 
[3] Bimbot, F., Bonastre, J.F., Fredouille, C., Gravier, G., Magrin-Chagnolleau, I., Meignier, S., Merlin, T., Ortega-Garcia, J., Petrovska-Delacretaz, D., Reynolds, D.: A tutorial on text-independent speaker verification. EURASIP Journal on Applied Signal Processing 2004(4), 430-451 (2004)

[4] Gajić, B., Paliwal, K.: Robust speech recognition in noisy environments based on subband spectral centroid histograms. IEEE Trans. Audio, Speech and Language Processing 14(2), 600-608 (2006)

[5] Paliwal, K.: Spectral subband centroid features for speech recognition. In: Proc. Int. Conf. on Acoustics, Speech, and Signal Processing (ICASSP 1998), Seattle, USA, vol. 2, pp. 617-620 (1998)

[6] Seo, J., Jin, M., Lee, S., Jang, D., Lee, S., Yoo, C.: Audio fingerprinting based on normalized spectral subband centroids. In: Proc. Int. Conf. on Acoustics, Speech, and Signal Processing (ICASSP 2005), vol. 3, pp. 213-216 (2005)

[7] Thian, N., Sanderson, C., Bengio, S.: Spectral subband centroids as complementary features for speaker authentication. In: Zhang, D., Jain, A.K. (eds.) ICBA 2004. LNCS, vol. 3072, pp. 631-639. Springer, Heidelberg (2004)

[8] Wu, X.: Optimal quantization by matrix searching. Journal of Algorithms 12(4), 663-673 (1991)

[9] Gersho, A., Gray, R.: Vector Quantization and Signal Compression. Kluwer Academic Publishers, Boston (1991)

[10] Reynolds, D., Quatieri, T., Dunn, R.: Speaker verification using adapted gaussian mixture models. Digital Signal Processing 10(1), 19-41 (2000)

[11] Rose, P.: Forensic Speaker Identification. Taylor \& Francis, London (2002)

[12] Chen, J., Huang, Y., Li, Q., Paliwal, K.: Recognition of noisy speech using dynamic spectral subband centroids. IEEE Signal Processing Letters 11(2), 258-261 (2004)

[13] Atal, B.: Effectiveness of linear prediction characteristics of the speech wave for automatic speaker identification and verification. Journal of the Acoustic Society of America 55(6), 1304-1312 (1974) 